\title{
\begin{tabular}{l|l} 
Mibraries & DSpace@MIT
\end{tabular}
}

\author{
MIT Open Access Articles
}

\section{Quasi-ballistic thermal transport from nanoscale interfaces observed using ultrafast coherent soft $x$-ray beams}

The MIT Faculty has made this article openly available. Please share how this access benefits you. Your story matters.

Citation: Siemens, Mark E. et al “Quasi-Ballistic Thermal Transport from Nanoscale Interfaces Observed Using Ultrafast Coherent Soft x-Ray Beams." Edited by Kong-Thon Tsen, Jin-Joo Song, Markus Betz, and Abdulhakem Y. Elezzabi. Ultrafast Phenomena in Semiconductors and Nanostructure Materials XV 7937 (February 2011): 793716 (c) 2011 Copyright Society of PhotoOptical Instrumentation Engineers (SPIE)

As Published: http://dx.doi.org/10.1117/12.871455

Publisher: SPIE

Persistent URL: http://hdl.handle.net/1721.1/113414

Version: Final published version: final published article, as it appeared in a journal, conference proceedings, or other formally published context

Terms of Use: Article is made available in accordance with the publisher's policy and may be subject to US copyright law. Please refer to the publisher's site for terms of use. 


\section{Quasi-ballistic thermal transport from nanoscale interfaces observed using ultrafast coherent soft x-ray beams}

Mark E. Siemens, Qing Li, Ronggui Yang, Keith A. Nelson, Erik H. Anderson, et al.

Mark E. Siemens, Qing Li, Ronggui Yang, Keith A. Nelson, Erik H. Anderson, Margaret M. Murnane, Henry C. Kapteyn, "Quasi-ballistic thermal transport from nanoscale interfaces observed using ultrafast coherent soft x-ray beams," Proc. SPIE 7937, Ultrafast Phenomena in Semiconductors and Nanostructure Materials XV, 793716 (21 February 2011); doi: 10.1117/12.871455 


\title{
Quasi-ballistic thermal transport from nanoscale interfaces observed using ultrafast coherent soft $x$-ray beams
}

\author{
Mark E. Siemens ${ }^{*}$,b, Qing Li ${ }^{\mathrm{b}}$, Ronggui Yang ${ }^{\mathrm{c}}$, Keith A. Nelson ${ }^{\mathrm{d}}$, \\ Erik H. Anderson ${ }^{\mathrm{e}}$, Margaret M. Murnane ${ }^{\mathrm{b}}$, and Henry C. Kapteyn ${ }^{\mathrm{b}}$ \\ a Dept. of Physics and Astronomy, Univ. of Denver, 2112 East Wesley Ave., Denver, CO 80208; \\ ${ }^{\mathrm{b}}$ Dept. of Physics and JILA, Univ. of Colorado, 440 UCB, Boulder, CO 80309 \\ ${ }^{\mathrm{c}}$ Dept. of Mechanical Engineering, 427 UCB, Boulder, CO 80309 \\ ${ }^{\mathrm{d}}$ Dept. of Chemistry, MIT Rm. 6-026, 32 Vassar Street, Cambridge, MA 02139 \\ ${ }^{\mathrm{e}} \mathrm{CXRO}$, Lawrence Berkeley National Laboratory, 1 Cyclotron Road Berkeley, CA 94720
}

\begin{abstract}
Heat dissipation from a nanoscale hot-spot is expected to be non-diffusive when a hot-spot is smaller than the phonon mean free path of the substrate. Our technique of observing diffraction of coherent soft x-ray pulses allows for very high resolution $(\sim \mathrm{pm})$ of thermally-induced surface distortion, as well as femtosecond time resolution of dynamics. We successfully model our experimental results with a diffusive transport model that is modified to include an additional boundary resistance. These results confirm the importance of considering ballistic transport away from a nanoscale heat source, and identify a means of correctly accounting for this ballistic transport.
\end{abstract}

Keywords: thermal transport, nanostructures, soft x-rays, ultrafast dynamics

\section{INTRODUCTION}

As the scale of functional, dissipative systems shrinks to significantly smaller than the mean-free-path $(\Lambda)$ of phonons in a material, thermal transport is no longer diffusive [1]. This transition to ballistic thermal transport has been experimentally demonstrated in a variety of nanostructures including superlattices [2], carbon nanotubes [3], and silicon nanowires [4]. In these cases of transport in a confined medium, the transition to ballistic transfer arises because phonon transport is limited by the nanostructure boundaries. In ballistic transport the phonon mean free path is limited to the structure size $L$ because phonon scattering occurs at boundaries [5]; this reduction in $\Lambda$ from the bulk value causes a decrease in the thermal conductivity $k=C v \Lambda / 3$.

Heat dissipation from a nanoscale hot-spot into a substrate - the nanoscale heat-sink problem - is of particular relevance to many technologies such as nanothermal therapy, thermoelectric conversion, and heat-sinking in microcircuits. Unlike ballistic transport inside of a nanostructure, decreasing the hotspot size does not directly shorten $\Lambda$ in the substrate. However, there may still be a significant ballistic effect due to the breakdown of Fourier/diffusive transport at length scales smaller than $\Lambda$ : the Fourier law for diffusive transport assumes local thermal equilibrium at every point, which requires phonon scattering events. When the source size is much smaller than $\Lambda$, this assumption is no longer valid because the Fourier law predicts temperature gradients that change (non-physically) over a length scale smaller than the phonon mean free path [6]. While some theory and modeling work has considered this problem [7,8], it has not been studied experimentally because of both the small length and short time scales involved. Past work using steady-state electrical measurements indicate small or zero nano-bulk ballistic effect [9].

In this work, we apply a new technique of time-resolved coherent soft x-ray diffraction to experimentally study this nanoscale heat-sink problem. In our experiment, nanopatterned nickel lines are impulsively heated by a near-infrared laser pulse from a Ti:Sapphire laser amplifier, and the thermal expansion and subsequent cooling are observed interferometrically, by monitoring the diffraction of a soft x-ray probe beam from the nanostructured surface. These coherent soft $\mathrm{x}$-ray pulses allow for very high resolution $(\sim \mathrm{pm})$ of surface distortion, as well as femtosecond time resolution capable of observing fast dynamics. We successfully model our experimental results with a diffusive transport

*msiemens@du.edu

Ultrafast Phenomena in Semiconductors and Nanostructure Materials XV, edited by Kong-Thon Tsen,Jin-Joo Song, Markus Betz, Abdulhakem Y. Elezzabi, Proc. of SPIE Vol. 7937, 793716 - (c) 2011 SPIE · CCC code: 0277-786X/11/\$18 - doi: 10.1117/12.871455 
model that is modified to include an additional boundary resistance. This resistance characterizes the error of the diffusive model and is proportional to the Knudson number $(K=\Lambda / L)$. These results confirm the importance of considering ballistic transport away from a nanoscale heat source, and identify a simple correction accounting for this ballistic transport.

\section{SAMPLE SPECIFICAITONS AND PREPARATION}

Samples providing a localized nanoscale heat source of a tunable size were required for a clear observation of the breakdown of the Fourier law for nano-bulk phonon transport. For this purpose, we prepared nickel nanoline samples on transparent substrates, as shown in Fig. 1. This configuration allowed for controlled and localized ultrafast heating; when exposed to a laser pump pulse, the transparent substrate was unaffected while the nickel lines absorbed the laser light, rapidly heating and then slowly cooling to the substrate. Experiments studying nano-bulk thermal transport away from a single wire, while technically possible using soft x-ray probing using dynamic Gabor holography, are experimentally challenging. For this reason, we chose to start with samples composed of a regular array of nickel lines with a duty cycle of $25 \%$ (linewidth $L$, center-to-center spacing $4 L$ ).

Periodic arrays of $20 \mathrm{~nm}$ high nickel lines were patterned with linewidths $L$ ranging from $65 \mathrm{~nm}$ to $2000 \mathrm{~nm}$ using electron-beam lithography and liftoff procedures. We prepared samples on two substrate materials in which we expected to see different transport phenomena: sapphire has a very long phonon mean free path $\left(\Lambda_{\mathrm{Sa}} \sim 120 \mathrm{~nm}\right.$ at room temperature) so non-diffusive transport was expected for nickel linewidths smaller than $\sim 100 \mathrm{~nm}$, while phonons in fused silica have a very short mean free path $\left(\Lambda_{\mathrm{FS}} \sim 1 \mathrm{~nm}\right.$ at room temperature) so diffusive transport is expected for all structures.
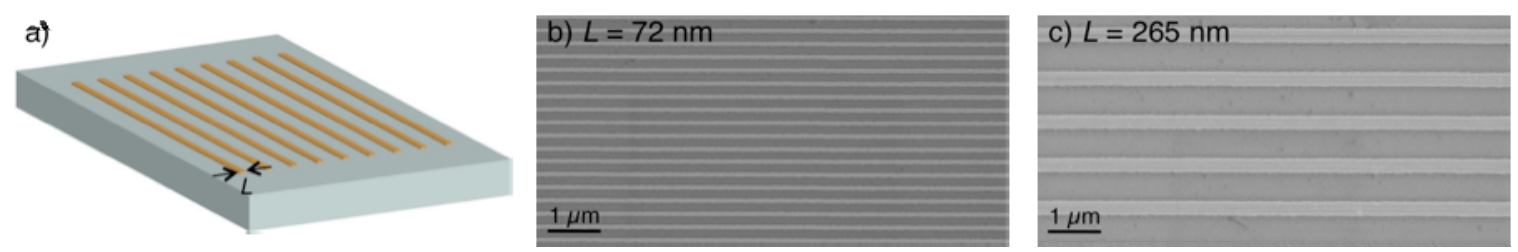

Figure 1: Sample configuration for one-dimensional localized nano-bulk thermal transport studies. a) Schematic of sample configuration, showing thin nickel nanolines on a transparent (sapphire or fused silica) substrate. b) and c) show SEM images of typical samples (lighter area is nickel).

\section{EXPERIMENT: OPTICAL PUMP, SOFT X-RAY PROBE}

Thermal transport away from the nickel nanolines was studied using a pump-probe technique shown in Fig. 2a, in which laser heating from ultrafast pulses at a wavelength of $800 \mathrm{~nm}$ was probed by even shorter soft x-ray pulses at a wavelength of $29 \mathrm{~nm}$. An ultrafast laser amplifier generating $2 \mathrm{~mJ}, 25 \mathrm{fs}$ pulses at a repetition rate of $2 \mathrm{kHz}$ and a wavelength of $800 \mathrm{~nm}$ was the source of both beams. Part of the $800 \mathrm{~nm}$ light from the amplifier was used as the pump pulse, which was sent through a mechanical time delay stage and then focused onto the sample for impulsive laser heating. The pump fluence of $2 \mathrm{~mJ} / \mathrm{cm}^{2}$ was in a relatively large spot size $700 \mu \mathrm{m}$ in diameter so that the probed area would be uniformly heated. The rest of the laser light from the amplifier was coupled into a hollow waveguide filled with Argon gas for high-order harmonic generation (HHG) of the $29 \mathrm{~nm}$ probe beam [10]. The resulting soft x-ray pulses were then focused onto the sample with a glancing-incidence toroidal mirror and a CCD camera recorded their diffraction from the grating formed by the nickel lines on the sample. 

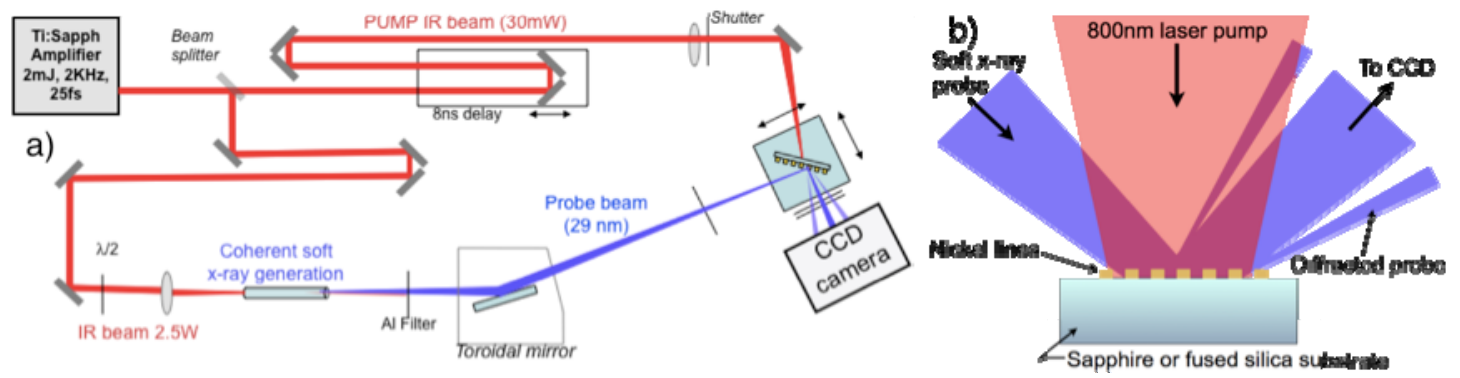

Figure 2: Experimental setup: a) Top view showing laser pulses from a laser amplifier split into pump and probe beams. The pump beam is focused onto the sample causing impulsive heating, while the probe beam is focused into a hollow waveguide filled with Ar gas for high-order harmonics generation. The resulting harmonics are focused onto the nanoline sample, and the resulting diffraction pattern is recorded by a CCD camera. b) Side view of pump and probe beams incident on sample and subsequent probe diffraction from the periodic nickel lines.

Unlike optical pump-probe measurements of thermal transport, where the signal is based on the temperature-dependence of the optical reflectivity [11], soft x-ray probe light is insensitive to thermal changes in the reflectivity of the sample. Instead, the signal we observe is due to thermal expansion and subsequent surface displacements; these interferometric measurements are possible because of the full spatial coherence of the soft x-ray light generated in the HHG process [10]. This change in the "height" of the nickel lines changes the interference condition between probe light reflecting from the lines vs the substrate surface, leading to a change in the diffraction intensity. One of the primary advantages of this interferometric technique is the short probe wavelengths involved: because we are using a wavelength $\sim 20 \times$ smaller than optical wavelengths, the technique is sensitive to surface displacements smaller than 1 picometer [12].

In the experiment, the delay time between pump and probe was stepped while diffraction spectra were recorded both when the pump beam was "on" and "off"; the result is a time dependent scan of the pump-induced change in diffraction. Scans were performed on samples with linewidths ranging from 65-2000 nm; typical results are shown for a variety of samples in Fig. 3. The soft x-ray diffraction difference scans have a few characteristic features in common: 1) a sharp rise at time $t=0$ indicating the arrival of the impulsive pump beam and subsequent photon-electron-phonon coupling that causes thermal expansion and an observed change in diffraction; 2) a decaying oscillatory component due to surface acoustic wave excitation and subsequent propagation in the sample [13]; and 3) a slow decay corresponding to the transfer of heat from the nickel nanowires into the substrate [14].

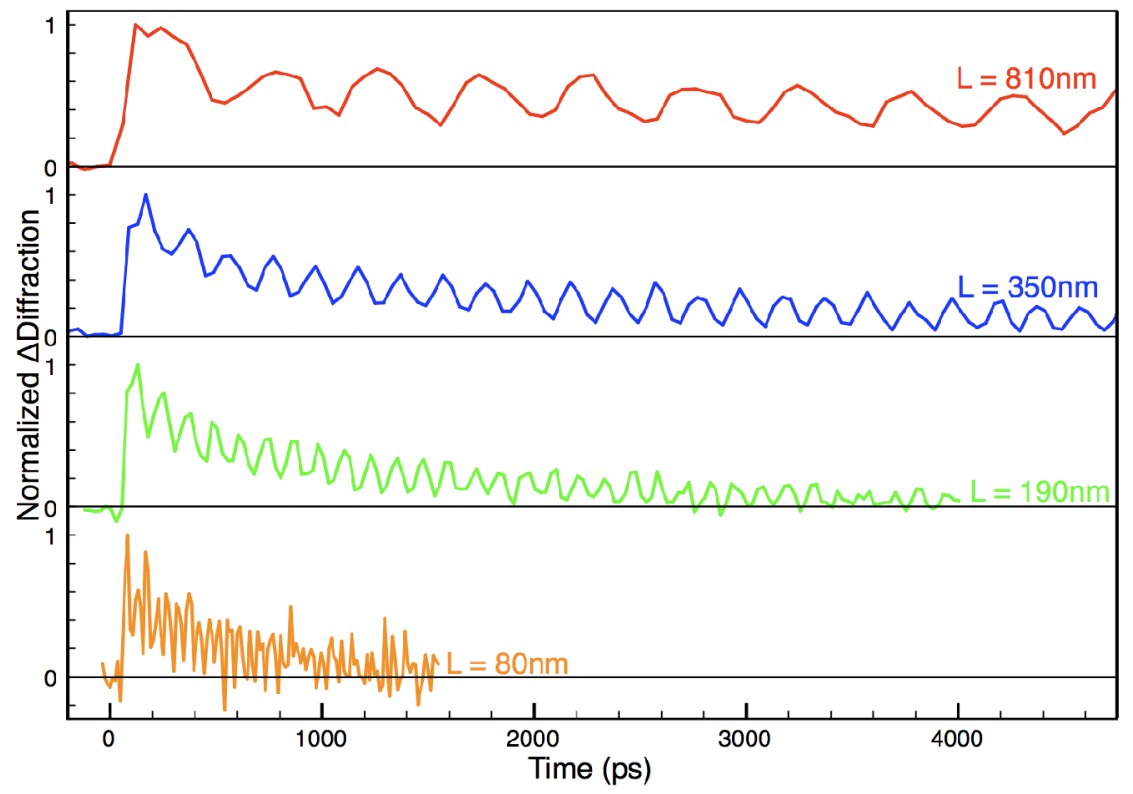

Figure 3: Experimental results, showing normalized change in diffraction as a function of pump-probe delay time for samples with a number of different linewidths. 


\section{RESULTS AND DISCUSSION}

We fit the dynamic diffraction difference data in Fig. 3 to a model including thermal diffusion and expansion and the subsequent effect on the soft x-ray diffraction pattern. The fits yield the effective interface resistance $r_{\text {Interface }}$, which includes the traditional thermal boundary resistance $r_{T B R}$ measured at thin film-substrate interfaces, as well as a ballistic correction component $r_{B C}$ accounting for any error in the diffusive Fourier law used to model the data; in general, $r_{\text {Interface }}$ $=r_{T B R}+r_{\text {Ballistic }}$. The ballistic correction component to the effective interfacial resistance is negligible when the Fourier law is valid (i.e. when $L>>1$ ), so $r_{\text {Interface }}=r_{T B R}$ for large linewidths or small mean free paths.

The results of the analysis for both fused silica and sapphire substrates are shown in Fig. 4. It is clear from Fig. 4a that no ballistic correction factor is needed to explain nano-bulk transport in a fused silica substrate; this is reasonable because $\Lambda_{F S} \sim 1 \mathrm{~nm}$, much smaller than the smallest structures used in these experiments. This is not the case in sapphire, where a significant correction factor is observed that grows larger for smaller linewidths; the long $A \sim 120 \mathrm{~nm}$ suggests that the Fourier law will break down for nano-bulk thermal transport away from nanolines with $L \sim 120 \mathrm{~nm}$, in keeping with the experimental data.
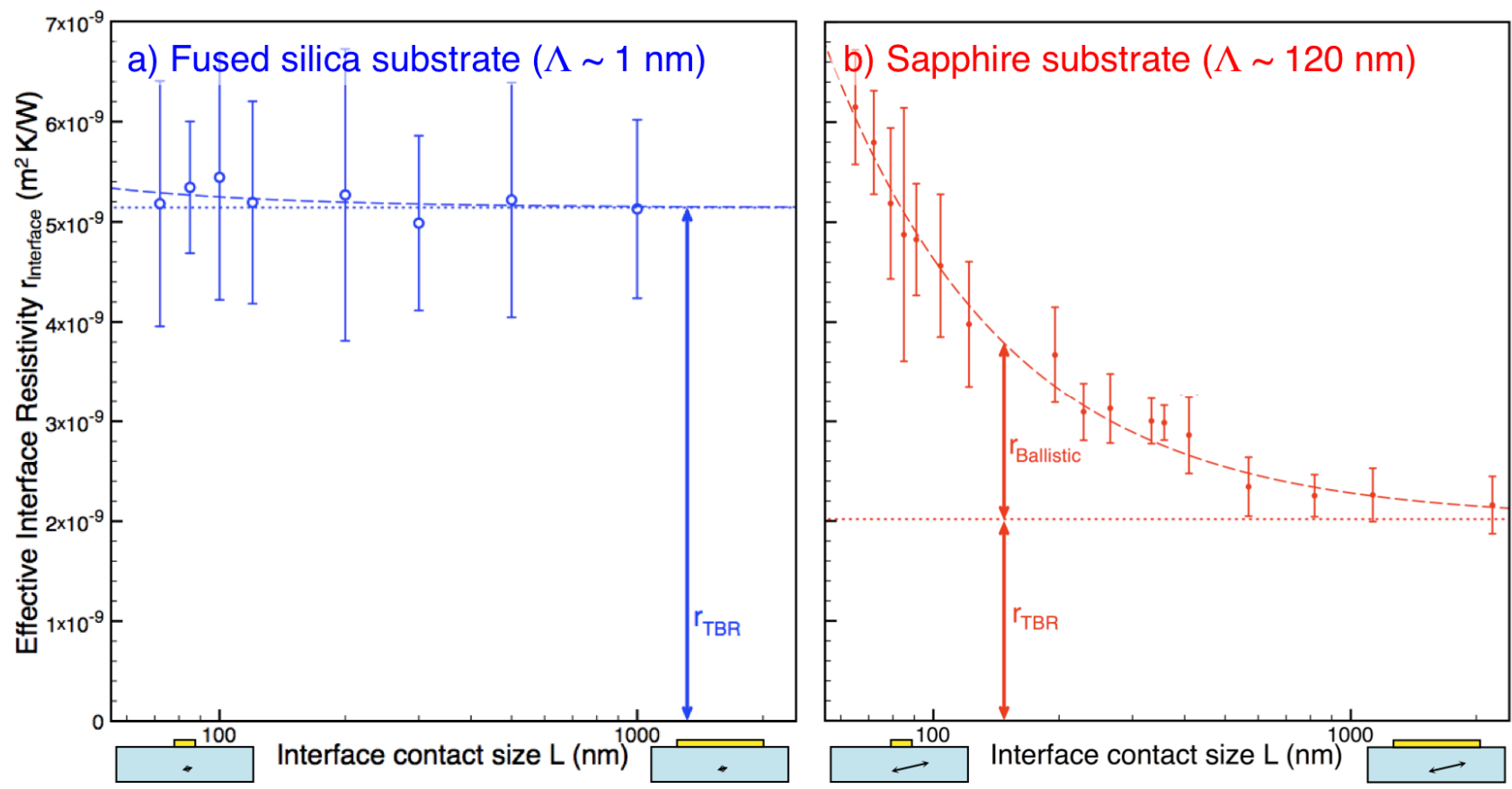

Figure 4: Measured effective thermal resistivity for nickel nanostructures of width $L$ deposited on a) fused-silica and b) sapphire substrates. The schematics below each figure indicate the change in linewidth on the scale of $\Lambda$ in the sample (indicated by the arrows). The blue and red dotted horizontal lines show the large-scale resistivity $r_{T B R}$ for data from the fused silica and sapphire substrates, while the dashed curves indicate $r_{\text {Ballistic }}$, the ballistic correction that must be included for linewidths $L \leq \Lambda$. The error bars indicate the standard deviation in the fits to the data.

Our measurements can be explained by a minimum heated region model (illustrated in Fig. 5), which suggests that thermal transport away from small hotspots can be explained by diffusive processes if the length scale of the heat source is considered to be larger than the phonon mean free path; i.e. $L_{\text {effective }} \rightarrow L+\Lambda$. This suggests that the ballistic correction resistance $r_{\text {Ballistic }} \Lambda / L$, which is the experimental dependence we observed for $\Lambda=120 \mathrm{~nm}$ in the red dashed line and $\Lambda=2 \mathrm{~nm}$ in the blue dashed line in Fig. 4. The fact that this ballistic correction factor matches the expected phonon mean free path in both materials is strong evidence for the minimum heated region model. Intuitively, this means that heat flow from a hotspot smaller than $\Lambda$ in the substrate should be understood as coming from a larger size equal to the phonon mean free path in the substrate. 


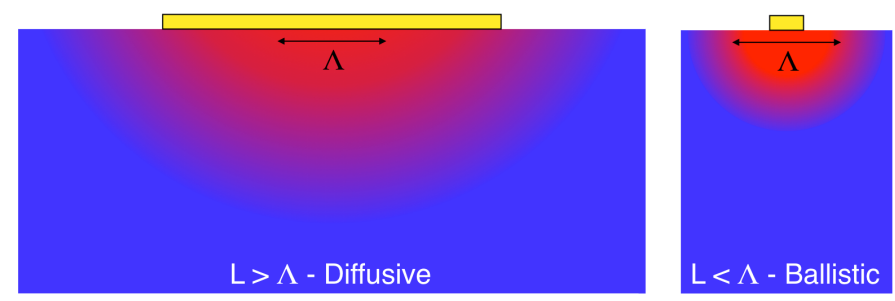

Figure 5: Illustration of nano-bulk thermal transport in the diffusive and ballistic cases. When the size nanoscale hotspot $L$ is larger than the phonon mean free path $\Lambda$, transport away from the hotspot is characterized by the hotspot size. However, when $L<\Lambda$, transport is characterized by the phonon mean free path length, independent of the hostpost size. This picture of nano-bulk transport is consistent with the minimum heated region model, and it specifies a limit on heat-sinking efficiency of small hotspots beyond the predictions of the Fourier law.

\section{CONCLUSION}

Using soft x-ray pulses as an ultrafast, interferometric probe, we perform the first direct and systematic measurement of quasi-ballistic nano-to-bulk thermal transport dynamics. We observe the breakdown of conventional Fourier heat conduction from hotspots smaller than the phonon mean free path in the substrate and demonstrate that an extra sizedependent resistance proportional to the Knudsen number $\Lambda / L$ is required to correct for non-diffusive transport phenomena unexplained by the Fourier law. In the future, these results should be extended to observe transport further into the ballistic regime by studying smaller structures or substrates with even longer phonon mean free paths $\left(\Lambda_{\text {Silicon }}=\right.$ $250 \mathrm{~nm}$ at room temperature). The results of these experiments and are important for reliability and performance design of nanodevices that generate heat, such as transistors, thermoelectrics, and photovoltaics.

\section{REFERENCES}

[1] Chen, G., Borca-Tasciuc, D., \& Yang, R. G. Nanoscale heat transfer. Encyclopedia of Nanoscience and Nanotechnology 7, 429-459 (2004).

[2] Capinski, W. S., Maris, H. J., Ruf, T., Cardona, M., Ploog, K., \& Katzer, D. S. Thermal-conductivity measurements of GaAs/AlAs superlattices using a picosecond optical pump-and-probe technique. Phys. Rev. B 598105 (1999).

[3] Chang, C. W., Okawa, D., Garcia, H., Majumdar, A., and Zettl, A. Breakdown of fourier's law in nanotube thermal conductors. Physical Review Letters, 101, 075903 (2008).

[4] Chen, R., Hochbaum, A. I., Murphy, P., Moore, J., Yang, P., and Majumdar, A. Thermal conductance of thin silicon nanowires. Physical Review Letters, 101105501 (2008).

[5] Joshi, A. A. and Majumdar, A. Transient ballistic and diffusive phonon heat-transport in thin-films. Journal of Applied Physics, 7431 (1993).

[6] Chen, G. Nonlocal and nonequilibrium heat conduction in the vicinity of nanoparticles. J. Heat Transfer 118, 539545 (1996)

[7] Chen, G. Ballistic-diffusive heat-conduction equations. Phys. Rev. Lett. 86, 2297-2300 (2001).

[8] Prasher, R. Predicting the thermal resistance of nanosized constrictions. Nano Letters, 5 2155-2159 (2005).

[9] Sverdrup, P.G., Sinha, S., Asheghi, M., Uma, S., \& Goodson, K.E. Measurement of ballistic phonon conduction near hotspots in silicon. Appl. Phys. Lett. 78, 3331-3333 (2001).

[10] Rundquist, A., Durfee, C.G., Chang, Z.H., Herne, C., Backus, S., Murnane, M.M., \& Kapteyn, H.C. Phase-matched generation of coherent soft x-rays, Science 280, 1412-1415 (1998).

[11] Cahill, D.G. Analysis of heat flow in layered structures for time-domain thermoreflectance. Rev. Sci. Inst. 75, 5119 (2004).

[12] Tobey, R., Siemens, M., Cohen, O., Murnane, M., Kapteyn, H., \& Nelson, K. Ultrafast extreme ultraviolet holography: dynamic monitoring of surface deformation. Opt. Lett. 32, 286 (2007).

[13] Siemens, M., Li, Q., Murnane, M., Kapteyn, H., Yang, R.G., Anderson, E., \& Nelson, K. High-frequency surface acoustic wave propagation in nanostructures characterized by coherent extreme ultraviolet beams. Appl. Phys. Lett., 94, 093103, (2009).

[14] Siemens, M., Li, Q., Yang, R.G., Nelson, K., Anderson, E., Murnane, M., \& Kapteyn, H., Quasi-ballistic thermal transport from nanoscale interfaces observed using ultrafast coherent soft X-ray beams. Nat. Mat., 9, 26, (2010). 